\title{
GESTIÓN DE LA CALIDAD UNIVERSITARIA: UNA VISIÓN DESDE LA EXPERIENCIA
}

\author{
Virginia Sánchez Molina* \\ Carlos Hernández Salazar**
}

Este artículo busca contribuir al estudio de la temática de la gestión de la calidad de una institución universitaria y sus programas. En la primera parte aborda los conceptos de calidad, liderazgo, evaluación, autoevaluación y acreditación, luego plantea los antecedentes y logros del proceso de desarrollo de la gestión de la calidad en la Universidad Nacional y concluye proponiendo los elementos de un sistema de gestión de la calidad.
The objective of this paper is to contribute with the study of quality management in a Higher Education institution and in its programs. The first part addresses the concepts of quality, leadership, evaluation, selfevaluation and accreditation. Then, we present the background of and achievements in the process ofdeveloping a high-level quality management at Universidad Nacional. Finally, the elements that must make up a quality management system are proposed.

* Máster en Educación Ambiental, Química, catedrática con formación y experiencia en el campo de la evaluación con fines de mejoramiento y acreditación. Fue Vicerrectora de Docencia de la Universidad Nacional (1995-1992), Vicerrectora de Extensión de la Universidad Nacional (1992-1989), y Directora de Extensión de la Facultad de Ciencias Exactas y Naturales de la Universidad Nacional (1989-1985). Actualmente se desempeña en el Programa de Evaluación Académica de la Vicerrectoría Académica de la Universidad Nacional y desarrolla varios proyectos de investigación en el área de gestión ambiental y gestión del recurso hídrico.

** Máster en Desarrollo Rural, agrónomo, con forrnación y experiencia en el campo de la evaluación con fines de mejoramiento y acreditación. Fue coordinador del área de proyectos productivos de la Vicerrectoría de Extensión. Actualmente se desempeña en el Programa de Evaluación Académica de la Vicerrectoría Académica de la Universidad Nacional. 


\section{Introducción}

La Universidad Nacional tiene como misión histórica la búsqueda de nuevos horizontes para el conocimiento y la formación de profesionales, que contribuyan con su quehacer a la transformación de la sociedad, hacia planos superiores de bienestar social y libertad. La universidad se constituye en conciencia crítica y creativa de la sociedad y promueve el desarrollo integral, autónomo, sostenible y equilibrado en el marco del respeto a los derechos humanos y la búsqueda del bienestar general en armonía con la naturaleza (Universidad Nacional, 1993).

La Universidad adquiere así un compromiso con la potenciación y la ampliación de oportunidades para los sectores sociales menos favorecidos, ofreciendo espacios para la formación de profesionales humanistas, con conciencia ambiental, críticos y propositivos y con la capacidad de generación de propuestas alternativas de solución ante las necesidades del desarrollo humano. Este compromiso institucional tiene como eje la renovación y mejora continua de los procesos académicos y de gestión institucional que contribuye a direccionar un nuevo modelo de desarrollo. En síntesis asume un compromiso de transformación del ser humano y su entorno a través del aprendizaje y la generación de conocimiento con calidad en un marco de responsabilidad social.

Así, la misión de la Universidad es un compromiso autoconferido y autodefinido que se establece desde dos grandes perspectivas, una relacionada con el pasado y el futuro (retrospectiva y prospectiva) y la otra tiene que ver con la mirada hacia adentro y hacia fuera de la institución. En las universidades antiguas la misión hacía referencia, esencialmente, acerca de lo realizado -el pasado- en las universidades jóvenes la misión apunta hacia el futuro, refiriéndose a las oportunidades, a los cambios, no buscan que se les juzgue por lo que son hoy.

Por otra parte, existen algunas universidades focalizadas hacia el entorno, responden a las demandas del mercado, su misión es una declaración efímera. Otras dirigen su atención hacia adentro, hablan de la sabiduría del conocimiento, están volcadas hacia sí mismas, se desentienden de su entorno, son autorreferentes. Ante estas diferentes formas de abordar su misión, el reto es encontrar el equilibrio entre ellas. De acuerdo con Atria (2002) una institución con liderazgo y calidad posee cabal comprensión de su encargo, de su comprọmiso, y de su misión.

En este sentido toda institución educativa aboga por la calidad, en su misión y principios se plantea la excelencia académica y la calidad. Todos dicen saber de calidad, pero pocos tratan de explicarla. Es un concepto como la belleza, percibirla es algo subjetivo, ponerla en acción es de sentido común, es difícil 
de definir, pero se reconoce cuando se está ante ella. Sin embargo, cuando hay deficiencias y fallas la responsabilidad siempre es de otro.

\section{La calidad}

Según Silva (2001) existen diferentes nociones de calidad:

- Calidad por excepción: conformarse con lo mejor, por ejemplo, la Universidad de Harvard, pero no todos pueden ni tienen que ser Harvard.

- Calidad como satisfacción de requisitos: cumplimiento de las competencias definidas.

- Calidad como satisfacción del usuario: cumplimiento de expectativas.

- Calidad como valor por dinero: excelencia a un precio aceptable, rentabilidad, rendición de cuentas ante los contribuyentes:

- Calidad como adecuación e idoneidad para lograr los propósitos: satisfacción del cliente, es decir de los alumnos, la sociedad, el gobierno, así como el cumplimiento de los objetivos declarados y los estándares adoptados.

La calidad institucional es objeto de modificación, y la tendencia normal es la búsqueda hacia niveles superiores. Aumentar los niveles de calidad implica tomar decisiones, implica la implementación de cambios e implica un cambio de actitud. Las decisiones y el cambio requieren fundamentación, sabiduría, requiere de una institución que aprende. En este marco la evaluación surge como una poderosa herramienta, que permite realizar una medición y un juicio valorativo en un marco referencial, es decir, con base en criterios y estándares que adoptamos para valorar cuánto y cámo realizamos lo que decimos que hacemos.

Alcanzarmayores niveles de calidad requiere, además, liderazgo, empoderamiento, participación por parte de los diferentes actores de la comunidad universitaria. Es esencial una disposición clara hacia el cambio, una actitud de compromiso y una visión compartida del cambio objetivo.

El liderazgo y la calidad de una universidad son una cualidad que se adquiere por reconocimiento de parte de los actores significativos para la institución:

- Otras universidades similares (prestigio entre sus pares).

- Oras instituciones de rango superior como los consorcios internacionales. redes internacionales, entre oras (sello de calidad por acreditación).

- Otras instituciones vinculadas como son las instituciones empleadoras de sus graduados, las instituciones que abren oportunidades para realizar abajos 
conjuntos (socias), las instituciones que contratan servicios (reconocimiento de competencias, reconocimiento de los títulos de los graduados).

- Personas vinculadas como los académicos, administrativos, gente que trabaja y que se forma en la universidad (reconocimiento de la identidad, del alma mater, se sienten parte de ella). El reconocimiento de todos estos actores debe ser explícito.

Así, el liderazgo institucional es el prestigio conferido por pares, es un sello de calidad regulada (acreditación), es la pericia demostrada por los graduados, es un plus de identidad, es decir, el liderazgo y la calidad es un sello que otros confieren a la institución.

En este sentido, encontramos instituciones o bien componentes de ellas, como por ejemplo, unidades, institutos, centros o programas académicos que son reconocidos por su calidad, y que si los analizamos encontramos que tienen una producción académica superior al promedio institucional, poseen múltiples y valiosas relaciones internacionales y nacionales, convenios y acuerdos de cooperación. Se caracterizan por la realización de trabajos en forma interinstitucional, cuentan con fuentes de ingresos adicionales al presupuesto institucional, y personal identificado y comprometido con el quehacer de la unidad, instituto o centro. En estos casos la calidad se percibe, se reconoce, aún cuando, en algunos de ellos, no existe un planteamiento explicito de cómo lograrla, simplemente han transitado hacia niveles superiores de calidad. En este marco, podemos decir de acuerdo con Silva (2001) que una institución universitaria o uno de sus programas educativos posee calidad si es capaz de demostrar que "progresa de manera continua y sistemática", empleando estrategias y recursos adecuados, hacia el "logro de sus objetivos" y compromisos y el "cumplimiento de criterios o estándares" establecidos.

La calidad universitaria involucra un conjunto de principios, estructuras y procesos, que le permiten fomentar y resguardar el mejoramiento continuo. La calidad está relacionada con la evaluación, la gestión y la acreditación.

\section{Antecedentes}

El desarrollo y la gestión de calidad es un proceso de crecimiento y maduración que, en la Universidad Nacional, tiene sus inicios de manera explícita y sistemática, a partir de experiencias con planes piloto de mejoramiento, como por ejemplo, el proyecto de mejoramiento de enseñanza de la química, así como la evaluación de programas y unidades específicas, la evaluación del desempeño de los profesores y la evaluación de proyectos académicos. 
De vital importancia, en este desarrollo de capacidad institucional para el desarrollo y la gestión de la calidad, resultó la participación de la UNA en proyectos internacionales como el Proyecto MHO: "Aseguramiento de la Calidad" y el Programa Columbus:

\begin{abstract}
"Autoevaluación con fines de mejoramiento", que permitieron la formación y actualización de académicos de la Universidad en estos nuevos campos relacionados con la evaluación y el mejoramiento de la calidad universitaria.
\end{abstract}

Un hito fundamental en este proceso fue la Reforma Académica, con su propuesta de evaluación generada internamente, denominada "EVA-UNA", concebido como "un proceso integral mediante el cual la Universidad evalúa, actualiza y reorganiza su quehacer académico, a la luz de su misión histórica, las tendencias del desarrollonacional e internacional, los avances disciplinarios, los requerimientos estudiantiles y las fortalezas institucionales, buscando elevar la calidad, la magnitud y el impacto de sus acciones en la sociedad" (1996, UNA.CSU-721-96).

Asimismo se inicia el proyecto de Gestión de la Calidad Institucional (20002004) y se desarrollan procesos de mejoramiento de la calidad de los servicios administrativos de apoyo a la academia: Departamento de Gestión Financiera, Registro, Proveeduría Institucional, Biblioteca, Departamento de Orientación Estudiantil; se concreta el proyecto de mejora del sistema de gestión de la información y la tecnología. También se inicia el proyecto de mejoramiento de la calidad de los proyectos de investigación y extensión.

En este marco de esfuerzo sistemático de la UNA, por alcanzar mayores niveles de calidad en su gestión en particular y en su quehacer en general se inician los procesos de autoevaluación de carreras con fines de mejoramiento y acreditación, logrando en un primer momento la acreditación por parte del SINAES de tres carreras, luego se da un fuerte impulso y son declarados procesos de prioridad institucional. Se impulsa y fortalece el proceso de evaluación del desempeño docente y se inicia un proceso de rediseño y actualización curricular con adecuación del ciclo lectivo. Acciones que en su conjunto ubican al quehacer docente y su gestión como eje central para el desarrollo y gestión de la calidad institucional.

\title{
La evaluación para el mejoramiento
}

La evaluación se da en dos momentos. En el primero se realiza un proceso de autoevaluación por parte de los actores involucrados en la acción que se evalúa -la carrera-. La evaluación es un mirarse en el espejo bajo dos 


\section{Así, el liderazgo institucional es el prestigio conferido por pares, es un sello de calidad regulada (acreditación), es la pericia demostrada por los graduados, es un plus de identidad, es decir, el liderazgo y la calidad es un sello que otros confieren a la institución.}

referentes: lamisión y objetivos que se ha propuesto y los estándares y criterios establecidos. Esto tiene sentido en la medida en que la evaluación se conceptualiza como una forma de conocer, aprender y relacionarse con la realidad para tratar de favorecer cambios para el mejoramiento. Es decir, el propósito fundamental de evaluares favorecer el aprendizaje por parte de los participantes acerca de las acciones que realizan, considerando sus sistemas de creencias, dado que cada uno percibe y construye la realidad desde sus vivencias, experiencias y conoci-
mientos, desde las creencias que posee y no de lo que está escrito en los documentos institucionales y es necesario clanificarlas y reconstruirlas para que emerja el cambio que deseamos hacia el mejoramiento (Sánchez y Alfaro 1997).

De esta manera, según Sánchez y Alfaro (1997) la evaluación se visualiza como un proceso continuo, integral, y participativo que permite identificar una problemática, analizarla y explicarla mediante información relevante, que proporciona juicios de valor para sustentar la toma de decisiones en una visión de mejoramiento continuo.

El segundo momento es la validación por pares externos del informe de autoevaluación que han elaborado los actores responsables de la carrera, con base en la lectura del mismo y una vista al campo para verificación. Esta segunda parte la realizan expertos externos a la institución, quienes elaboran su informe para un ente acreditador, cuando el proceso se realiza para este fin.

\section{La autoevaluación}

Por la importancia comprobada y el potencial que revisten los procesos de autoevaluación en el mejoramiento del nivel de calidad de las carreras y su potencial para inducir dinámicas de cambio en todos los ámbitos de la institución, se amplía al respecto en este apartado.

La autoevaluación se puede definir como un proceso participativo de reflexión y autoestudio, de análisis y contrastación de las acciones que se desarrollan en el contex to universitario y cómo estas concuerdan con la misión, principios, valores institucionales y con los estándares establecidos por la academia y los gremios de profesionales en lo que a ellos corresponda (Sánchez y Alfaro, 1997).

El proceso de autoevaluación parte del supuesto de que está orientado hacia la toma de decisiones, sabiendo que esta toma de decisiones está determinada 
por factores personales, políticos y culturales, por lo que requiere de la participación y el compromiso de las autoridades tanto de la unidad académica como de la facultad y las autoridades superiores de la Universidad. El proceso de autoevaluación debe contar con la participación de todos los responsables de la acción que se evalúa, los actores directos: el personal docente, estudiantes y funcionarios administrativos; los actores indirectos: los egresados, empleadores, gremios, y academias. La participación de los actores no consiste en contestar encuestas o entrevistas, sino en el análisis y valoración de los resultados obtenidos en relación con los criterios y parámetros establecidos por la institución y el ente acreditador cuando corresponda. Así el propósito fundamental de evaluar es favorecer el aprendizaje por parte de los participantes acerca de las acciones que realizan, considerando sus sistemas de creencias y establecer consensos para la implementación de acciones de mejoramiento. El proceso de autoevaluación nos dice:

¿Qué somos?

¿Por qué lo hacemos?

¿Cómo se relaciona lo que

hacemos con el por qué lo hacemos?

Objetivos vs actividades y resultados

¿Qué tan bien lo hacemos?

Calidad, efectividad, equidad

¿Con cuánto lo hacemos?

$¿$ ¿Hace diferencia si lo hacemos o no?

¿Cómo podemos mejorar lo que hacemos?
Actividades y funciones

Misión y objetivos
Recursos, eficiencia

Pertinencia, trascendencia impacto

Planificación, aprendizaje, cambio, planes de mejoramiento

En el proceso de autoevaluación de una carrera se evalúan los siguientes aspectos:

- Personal académico

- Desarrollo del currículo

- Estudiantes

- Infraestructura y recursos

- Administración, servicios y apoyo

- Resultados e impactos 
La autoevaluación parte de la academia, ubica la carrera como eje central, tanto para el proceso de autoevaluación como para la implementación de los cambios y el mejoramiento, desde una visión integral y sistémica, como se visualiza en la Figura $\mathrm{N}^{\circ} 1$. Considera los recursos humanos: el personal académico, administrativo y estudiantes, como elemento esencial para el logro de las metas propuestas. Tiene como referente la misión, objetivos y metas de la unidad académica a la que pertenece la carrera, así como la misión y objetivos institucionales. En concordancia con lo planteado en los párrafos precedentes, toma en cuenta el entorno: país, comunidad, gremios, egresados, empleadores, el avance de la disciplina. Valora la gestión institucional que involucra:

a) Los servicios de apoyo, tanto a estudiantes por parte del registro, biblioteca, vida estudiantil, financiero, transportes, suministros, y de la unidad académica a la que pertenece la carrera, la coordinación de la carrera; como a los funcionarios por parte de recursos humanos, financiero, proveeduría, servicios generales, etc; asimismo, los servicios a usuarios externos, padres de familia, estudiantes potenciales y público en general.

b) La gestión de las autoridades de la unidad y la facultad.

c) La gestión de las autoridades superiores.

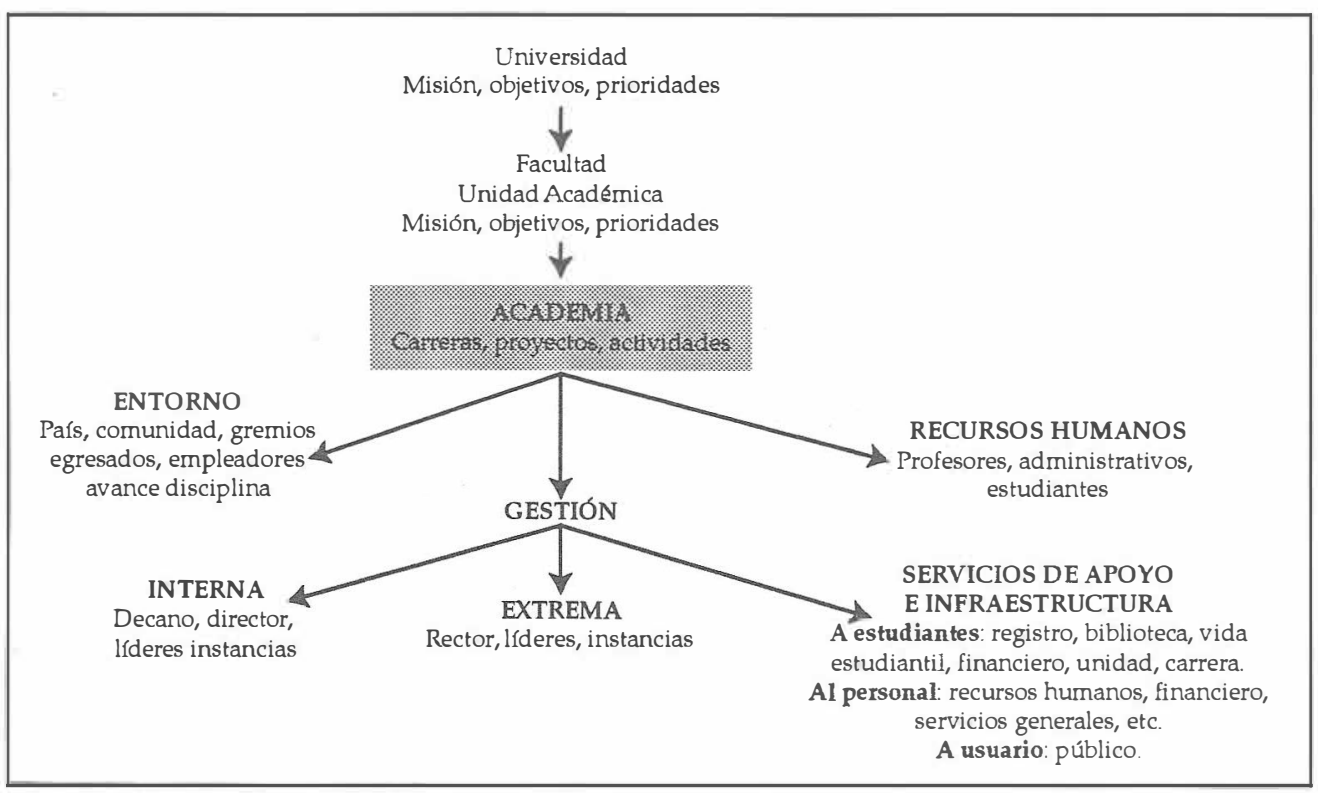

Figura $\mathbf{N}^{\circ}$ 1. Elementos fundamentales de un proceso de autoevaluación. 
El proceso de autoevaluación culmina con un Informe Final de Autoevaluación, que incluye un plan de mejoramiento para superar las debilidades encontradas, el cual debe estar incorporado en el Plan Operativo Anual (POA) de la Unidad Académica, el POA de la Facultad y el POA Institucional, explicitando las actividades, metas, indicadores de logro, responsables, cronograma y recursos.

El plan de mejoramiento puede incluir la necesidad de mejoras no solo en la unidad a la que pertenece la carrera, sino en otros niveles e instancias, por ejemplo, las unidades de servicio y apoyo administrativo, facultad, políticas y procedimientos institucionales, entre otros. Se establece así la relación entre la evaluación, la toma de decisiones, la planificación y la gestión y el control, no solo para la docencia, para la carrera autoevaluada, sino para toda la gestión institucional.

De esta manera a partir de la autoevaluación de una carrera se valora y retroalimenta indirectamente todos los servicios e instancias de la universidad, es decir, la gestión para atender la formación de los estudiantes, para lograr la misión institucional, para lograr la calidad y el reconocimiento del liderazgo institucional por parte de los actores significativos como se planteó al inicio de este documento.

\section{La acreditación}

La acreditación es el reconocimiento formal y público de que una institución o uno de sus programas está cumpliendo satisfactoriamente con los objetivos declarados y con un conjunto de referentes establecidos: criterios y estándares de calidad (Silva, 2001).

La acreditación tiene impacto en los liderazgos institucionales, en un modelo de acreditación pública con autoevaluación y juicio de expertos externos, el impacto se da en todos los actores significativos, toca la vida institucional, al personal y estudiantes de la universidad; significa que lo que se hace se está haciendo bien y además se ha trazado un camino y se ha adquirido un compromiso para seguir haciéndolo cada vez mejor.

Una institución que muestra su liderazgo hacia afuera, por ejemplo, con la acreditación, debe tenerlo hacia adentro, el punto no es únicamente tener el sello de acreditación. Es indispensable no perder de vista el espíritu, el fondo del asunto, de ahí el valor que tiene la mirada hacia dentro de cada uno de nosotros mismos, es ese "mirarse en el espejo" a través del proceso de autoevaluación; esta es la parte más rica del proceso, lo que nos va a hacer cambiar, y que se refleja en creer en nosotros mismos, en amar lo que estamos haciendo. Lo importante no es solo el reconocimiento público, la acreditación, sino cómo nos impacta, cómo nos comprometemos con el mejoramiento y cómo vamos a avanzar en una gestión de calidad. 


\section{Resultados y consecuencias de los procesos de autoevaluación con fines de mejoramiento y acreditación en la UNA}

La literatura relacionada con autoevaluación, evaluación y acreditación de carreras reporta importantes aportes y efectos en el mejoramiento de la calidad de las carreras en un primer nivel y la dinamización de procesos de cambio y mejoramiento en otros ámbitos de la gestión universitaria, tanto en el plano académico como en el de los servicios de apoyo a la academia

De manera general se aprecian mejoras e implementación de "buenas prácticas" en los siguientes aspectos a nivel de carrera y unidad académica:

- Desarrollo de capacidad para realizar procesos de autoanálisis/ aprendizaje.

- Implementación de nuevas y mejores prácticas de archivo.

- Cultura de sistematización de información.

- Planificación y gestión curricular.

- Cultura de evaluación.

- Plan de mejoramiento / reacreditación.

- Certificación pública de la calidad de la carrera o institución.

- Reconocimiento del título que se ofrece .

- Prestigio/. Disposición y compromiso para el cambio y el mejoramiento/. Disposición del personal a ofrecer servicios de calidad.

- Capacitación.

- Equipamiento.

- Imagen y limpieza.

- Infraestructura.

A nivel del contexto se evidencian logros en cuanto a:

- Un reconocimiento hacia el personal de las carreras.

- La oportunidad de ofrecer asesorías en el campo.

- La posibilidad de modificar políticas de nombramientos en el Servicio Civil.

- Un reconocimiento en el mercado.

A nivel institucional:

- Se ha logrado la priorización en la asignación de recursos, definiendo como prioridad a las unidades que realizan y culminan un proceso de autoevaluación con fines de mejoramiento y acreditación y se comprometen en la ejecución 
de un plan de mejoramiento (redistribución presupuestaria, rompimiento de la asignación presupuestaria histórica).

- Se avanza en la adquisición de un sistema de información institucional que brinde información oportuna y confiable para sustentar la toma de decisiones y la realización de los procesos de autoevaluación.

- Mejoras en:

- Procesos de matrícula y registro.

- Gestión de información.

- Algunos servicios.

\section{Gestión de la calidad}

De acuerdo con lo anterior la gestión de la calidad se basa en los siguientes criterios:

- Liderazgo organizacional y de responsabilidad pública

- Planificación estratégica y desarrollo

- Focalización en el alumno, sus necesidades y expectativas

- Administración del proceso educativo y de apoyo, es decir, desarrollo curricular: diseño, gestión y evaluación curricular con el soporte administrativo

- Focalización en los profesores, profesores que saben lo que enseñan, aman lo que enseñan, aman la enseñanza y creen en quienes enseñan

- Información y análisis que sustente la toma de decisiones

- Medición y valoración, es decir, evaluación sistémica, insumo, proceso, producto con referencia a un conjunto de estándares

- Toma de decisiones de cambio

- Resultados: efectividad y satisfacción

- Utilidad que se reconoce en los resultados de la evaluación en la implementación de las acciones correctivas (credibilidad)

- Implementación de soluciones con los actores (participación y concreción)

La gestión de calidad requiere el liderazgo y compromiso de las autoridades en los diferentes niveles, el perfeccionamiento y capacitación de los funcionarios, la participación de los académicos, estudiantes y administrativos en los procesos, la reflexión sobre los resultados obtenidos, el currículum centrado en el estudiante en función de formar un egresado con un perfil profesional de utilidad para la sociedad (responsabilidad social) y la voluntad de implementar las decisiones de cambio. 


\section{Sistema de Calidad Institucional}

Se entiende que una institución tiene calidad en el tanto cuenta, de manera sostenida, con una estructura efectiva para alcanzar la misión y la visión propuesta, con sistemas eficaces y transparentes en la toma de decisiones, con altas competencias y confiabilidades en los gestores y con personas cuyos talentos, valores y acciones se potencian para alcanzar la misión institucional. De acuerdo con la experiencia, la Universidad Nacional posee la madurez y capacidad para establecer un sistema de calidad centrado en la academia, que coordine e interrelacione las acciones de mejoramiento de la calidad en el ámbito de los servicios administrativos y de apoyo, de la gestión institucional, la planificación estratégica y la oferta académica.

Se propone así un Sistema de Gestión de la Calidad en la UNA, como un esfuerzo organizado y sistemático, para alcanzar mayores niveles de calidad en la gestión institucional y en su quehacer en general, que permita tomar decisiones e implementar las acciones y los cambios necesarios para lograrlo. Dicho sistema tendrá como eje la academia y a partir de él involucra toda la acción universitaria.

Se propone que dicho sistema de calidad considere los siguientes elementos (ver Figura $\mathrm{N}^{\circ}$ 2):

a- Compromiso y política: parte de la definición de una política y lineamientos institucionales, aprobada y compartida por todos los actores, elaborada a partir de un diagnóstico existente producto de los procesos de autoevaluación con fines de mejoramiento y acreditación.

b- Planeamiento: un sistema que explicite el compromiso con la calidad y el mejoramiento continuo a partir de objetivos y metas con indicadores de desempeño.

c- Implementación: un sistema:

i. que defina, realice y documente las acciones de sensibilización, capacitación y comunicación pertinentes para contar con los recursos humanos formados y motivados para avanzar en el mejoramiento de la calidad.

ii. que establezca los procedimientos operacionales y estén a disposición de todos para desarrollar las acciones y lograr los objetivos y metas propuestas.

iii. que establezca los controles y el monitoreo y los documente.

iv. que realice las revisiones, evaluaciones que le permitan detectar las debilidades y mejoras pertinentes, así como la revisión de las políticas propuestas y su continuo mejoramiento. 


\section{ELEMENTOS SISTEMA DE GESTIÓN DE LA CALIDAD}

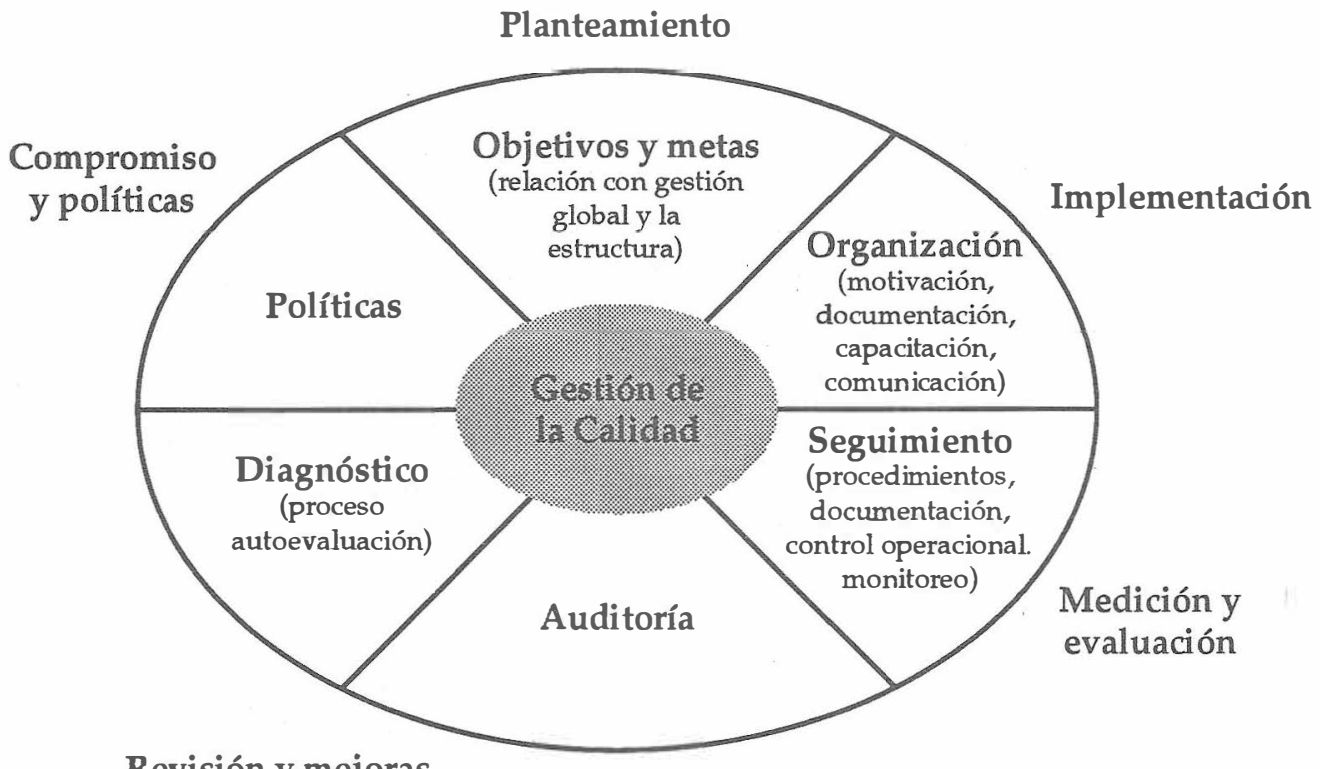

Revisión y mejoras

Podemos concluir que la gestión de la calidad es un proceso que se sustenta en la evaluación para la toma de decisiones para el mejoramiento, requiere de la planificación para organizar los cambios, de la gestión ponerlos en marcha y del control como la acción político-adminis rativa que vela porque los cambios propuestos e implementados en las políticas, lineamientos y normativa se estén ejecutando. Un sistema que interrelaciona y coordina las acciones de evaluación, planificación, gestión y control para la gestión de la calidad.

\section{CALIDAD}

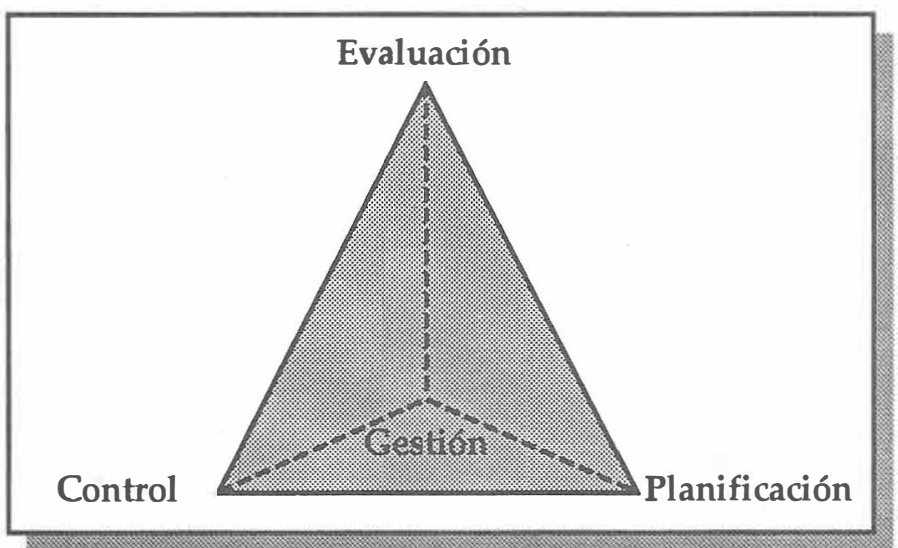




\section{Referencias}

Atria, R. (2002). La acreditación y su relación con el liderazgo institucional. Seminario la formación de profesores desde las humanidades y los temas que influyen hoy: estándares de desempeño, autoevaluación y acreditación. Santiago de Chile. 2002.

Sánchez, V. y Alfaro, G. (1997). Informe final Proyecto de autoevaluación, Programa Columbus. Seminario Internacional Proyecto Alfa/Columbus. Heredia, Costa Rica.

Silva, M. (2001). Gestión Calidad y Evaluación Externa. Taller internacional de capacitación de potenciales coordinadores de equipos de pares académicos para la evaluación externa del SICEVAES.

Universidad Nacional (1993). Estatuto orgánico. Heredia, Costa Rica. 